\title{
Evaluation and simple modification of the gastrin test
}

\author{
M. C. MASON ${ }^{1}$ AND G. R. GILES \\ From the University Department of Surgery, The General Infirmary, Leeds
}

Investigations of gastric acid secretion have always been a somewhat arduous experience for the patient. Apart from the physical discomfort of nasogastric intubation and subsequent enforced immobility, many of the problems stem from the side effects of the gastric stimulant. A histamine infusion (Lawrie, Smith, and Forrest, 1964) is less unpleasant than the standard augmented histamine test (Kay, 1953) but is too complex to obtain widespread clinical acceptance.

The recent general availability of pentagastrin (Peptavlon-I.C.I.) with its minimal attendant side effects (Multicentre Pilot Study, 1967; Mason, Giles, and Clark, 1969) for use instead of histamine should, however, result in a further lessening of the discomforts. The present problem is to formulate a simple and efficient test which can be recommended to the busy, practising clinician.

Johnston and Jepson (1967) suggested an intramuscular technique which utilized the prompt onset of gastric acid stimulation which follows such an injection of pentagastrin. As this test is concluded only 30 minutes later, it is dependent upon the peak of secretion having been achieved within that interval. Should the peak be delayed in any patient, the accuracy of the test would be impaired.

The present study was designed to determine whether the peak secretion occurred within the 30 minutes and so to assess the validity of the test in its present form. A comparison was also made with the response which followed a pentagastrin infusion (Mason et al, 1969), the routine method of estimating maximal acid secretion in this gastric laboratory.

\section{MATERIALS AND METHODS}

INJECTION OF PENTAGASTRIN Of the 33 patients with intact gastric vagal innervation submitted to testing, 17 suffered from peptic ulceration, 11 from colitis, two from cholecystitis, and one from a hiatus hernia. One more had previously undergone a partial gastrectomy with a Polya type of anastomosis and a further case was subsequently shown to have widespread carcinomatosis from an unsuspected colonic primary.

${ }^{1}$ In receipt of a full time Medical Research Council award.
After an overnight fast, a 14 FG nasogastric tube was passed and manoeuvred into a suitable position without radiological control. Resting juice was removed for 15 to 20 minutes and then discarded. In addition, although not included in the technique of the test as outlined by Johnston and Jepson, the spontaneous secretion was collected for 30 minutes, to reduce the possibility of missing a case of the Zollinger-Ellison syndrome and also because it has been the policy of this unit to record the level of 'basal' secretion. (This collection of spontaneous secretion is not recommended as part of the routine in the general use of the gastrin test.)

Pentagastrin, $6.0 \mu \mathrm{g} / \mathrm{kg}$, was then given as a deep intramuscular injection. (This was done personally by M.C.M. on all occasions to reduce the possibility of a subcutaneous injection being given in error.) Subsequent secretions were collected in 10-minute samples for a further $\mathbf{4 0}$ minutes. The juice was filtered through glass wool, the volume measured, and the acidity estimated by the titration of a $1 \mathrm{ml}$ aliquot with $\mathrm{N} / 100$ sodium hydroxide solution using Töpfer's reagent as the indicator. Results were therefore derived as milliequivalents of acid per 10-minute sample.

All the usual precautions were taken to maintain the patency of the nasogastric tube and to encourage good recovery of secretions.

INFUSION OF PENTAGASTRIN Thirteen of the above patients also had a pentagastrin infusion test using a dose of $1.2 \mu \mathrm{g} / \mathrm{kg} / \mathrm{hr}$ (Mason et al, 1969). The infusion was continued for one hour and the collections were made in 15-minute samples. The acid secreted per sample were similarly estimated and the maximal output was derived by doubling the peak 30-minute secretion. Intramuscular and intravenous tests were usually performed on different days. However, if the tests were carried out on the same day the intramuscular test was not started until at least an hour had elapsed after the end of the infusion. This allowed ample time for the secretion to return to basal levels (Mason, 1968).

\section{RESULTS}

INJECTION OF PENTAGASTRIN Figure 1 shows that maximal secretory rates were swiftly attained but also that there is no significant difference in acid output between the second and fourth 10-minute periods. The overall mean maximal secretion was achieved in the third 10 -minute period. This was 


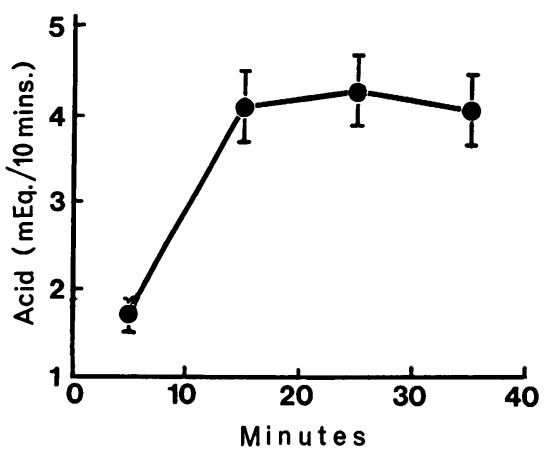

FIG. 1. Acid output following intramuscular pentagastrin $6.0 \mu g / k g($ mean $+S E M)$.

not significantly greater than either the 10 minute period preceding or succeeding it $(P=<0 \cdot 3)$.

Johnston and Jepson (1967) suggested that the secretion collected from the tenth to the thirtieth minutes should be used as the basis for calculation of the maximal response, as they claimed that the peak response could be relied upon to have occurred within that interval. Table I shows that although this was true in 15 of the 32 tests, in the remaining 17 , a higher level of secretion was obtained between the twentieth and fortieth minutes. In a single case

\section{TABLE I}

THE DISTRIBUTION, IN TIME, OF THE PEAK ACID SECRETION AFTER PENTAGASTRIN IM $6.0 \mu \mathrm{g} / \mathrm{kg}$

\begin{tabular}{l} 
Number of Patients \\
\hline Expected Observed \\
(Johnston and \\
Jepson, 1967)
\end{tabular}

Peak

\begin{tabular}{llrl}
\hline 10 to $30 \mathrm{~min}$ & & 33 & 15 \\
20 to $40 \mathrm{~min}$ & & 0 & 17 \\
& Total & 33 & $32^{1}$
\end{tabular}

${ }^{1}$ Excludes one patient in whom the outputs were the same in both periods.

the output was the same for these two periods of collection. These findings differ significantly from those which could be predicted from the data presented by the original authors $\left(\chi^{2}=6.21\right.$, $\mathrm{n}=1, \mathbf{P}=<0.02)$. The differences between the maximal acid secretions calculated from the 10-to-30 and the 20-to-40-minute periods are shown in Figure 2. This again illustrates that the peak response frequently does occur within 30 minutes of the pentagastrin injection.

The 20-minute period with the highest acid secretion, no matter where it occurs, is referred to as the 'optimal' 20-minute secretion. When this

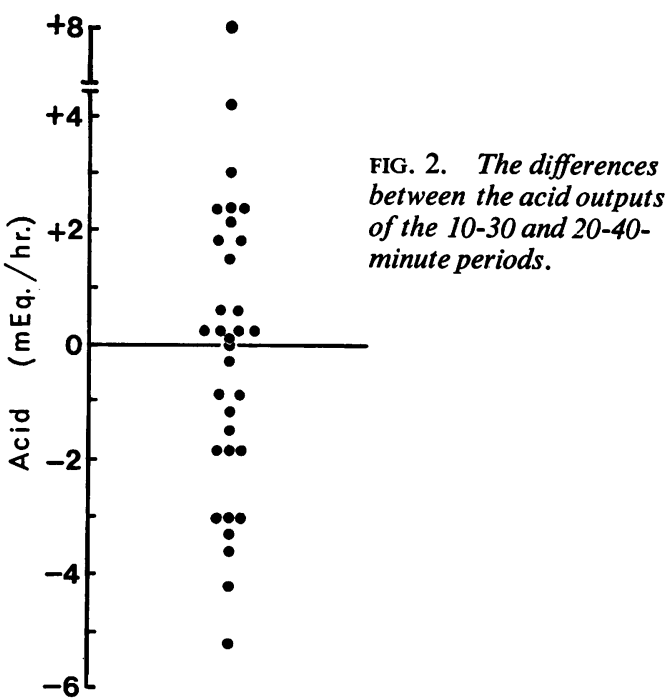

optimal secretion is used to calculate the maximal response, the resulting values are significantly greater than when Johnston and Jepson's method of calculation is employed $(\mathrm{t}=3.43, \mathrm{P}=<0.01)$.

INFUSION OF PENTAGASTRIN After the intravenous infusion a mean maximal secretion of $32.0 \pm 3 \cdot 2$ was obtained. For the same group of patients the mean maximal secretion was $27.0 \pm 2.9 \mathrm{~m}$-equiv/hr when the intramuscular test was used and Johnston and Jepson's method of calculation was followed. The latter gave a response which was $83.9 \%$ of that which followed the intravenous infusion. This difference is of statistical significance $(t=3 \cdot 2, P=$ $<0.01$ ).

When the optimal 20-minute secretion is used to calculate the maximal response of the intramuscular test, the mean maximal output is increased to $27.8 \pm 2.9 \mathrm{~m}$-equiv/hr and to $86.3 \%$ of the intravenous infusion. Figure 3 shows that by using this method of calculation the correlation coefficient (r) is also improved from 0.874 to 0.913 and the standard error of estimate (SEE) from \pm 4.9 to $\pm 4 \cdot 1$ m-equiv.

\section{DISCUSSION}

The claimed advantages of the gastrin test are its brevity, simplicity, and lack of side effects. All these claims can be supported, but it is felt that the test, as originally presented, has been unnecessarily abbreviated with consequent detriment to its accuracy.

If any studies of gastric acid secretion are to 


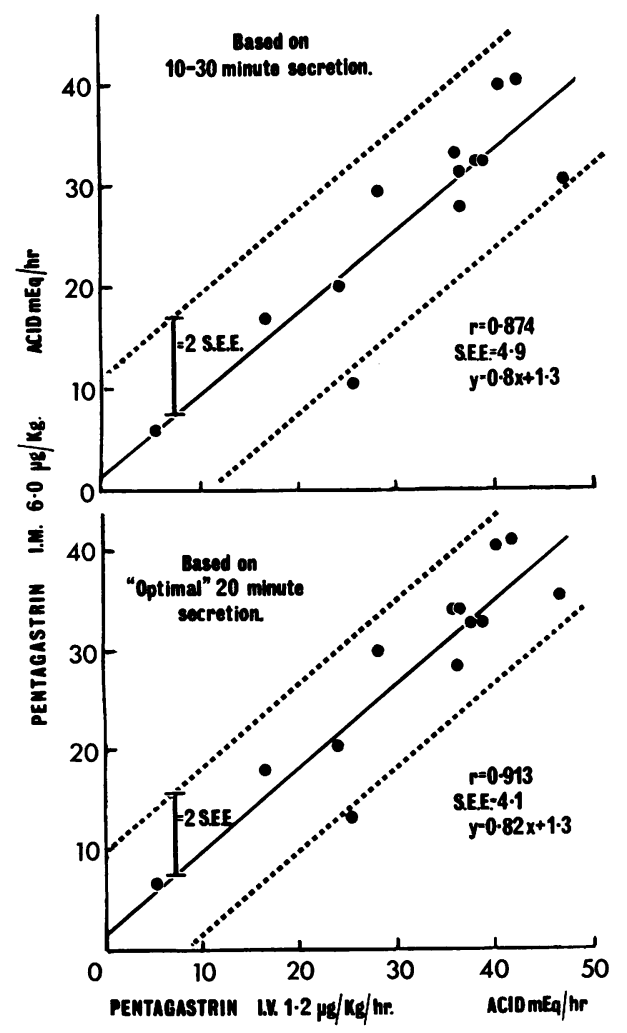

FIG. 3. Comparisons of maximal acid response after intramuscular and intravenous pentagastrin.

yield worthwhile information, diligence and experience on the part of the person conducting the test are essential. It cannot be too strongly emphasized that these investigations should not be supervised solely by a busy ward nurse. To obtain reliable results adequate collection of secretions is essential and this in turn depends upon suitable placing of the nasogastric tube and positioning of the patient. All of these preliminaries take time but are necessary for the correct and accurate performance of the test. To extend the test by 10 minutes more than the original authors recommended, therefore, does not seriously prolong the overall length of the test or materially increase the discomfort for the patient.

It has been clearly shown that the peak response of gastric acid secretion does not always occur within 30 minutes of an intramuscular injection of pentagastrin $6.0 \mu \mathrm{g} / \mathrm{kg}$. If this had been so, there should have been a significantly smaller acid output in the 20-to-40-minute period than in the 10-to-30-minute period, which was not the case.

No practical difficulties accrue from extending the test for an extra 10 minutes. It is, therefore, recommended that the test should be performed in the manner proposed by Johnston and Jepson (1967) except that, after the pentagastrin injection, there should be four 10-minute collections of acid secretion. The acid output of the original optimal 20-minute secretion should be determined and multiplied by 3 to express maximal acid secretion as milliequivalents per hour. Allowance is thus made for those patients whose peak rate of acid secretion is achieved somewhat later than had originally been supposed. This simple modification maintains the advantages of the original test and also has been shown to give superior results.

When the intramuscular and intravenous infusion techniques are compared it has been shown that the latter gave a significantly greater response. The $86.3 \%$ of the intravenous infusion response which follows the intramuscular administration of pentagastrin is of the same order of magnitude as has been reported when histamine has been given by the two differing routes (Lawrie et al, 1964; Lawrie and Forrest, 1965).

\section{SUMMARY}

The gastrin test (Johnston and Jepson, 1967) has been used to estimate the maximal secretory capacity of the human stomach. It was not possible to confirm that the peak 20-minute response always occurred within 30 minutes of the pentagastrin injection. Consequently, it is recommended that secretions are collected for $\mathbf{4 0}$ minutes and that the peak 20-minute output be used as the basis for calculation of the maximal secretion. This simple modification gives an improved correlation with the values obtained from an intravenous infusion.

In this form, the intramuscular test evokes a response approximately $85 \%$ of that which follows an intravenous infusion and is probably the 'gastric test' of choice for the practising clinician.

We thank Professor J. C. Goligher for his interest in this work, Dr. J. D. Fitzgerald, of I.C.I., Pharmaceuticals Division, for the gift of pentagastrin, and Mr. Iain Gillespie of the University Department of Surgery, Western Infirmary, Glasgow, for his helpful comments and guidance. The technical assistance of Miss $\mathbf{S}$. Halloway and Miss S. Basham is gratefully acknowledged.

\section{REFERENCES}

Johnston, D., and Jepson, K. (1967). Use of pentagastrin in a test of gastric acid secretion. Lancet, 2, 585-588.

Kay, A. W. (1953). Effect of large doses of histamine on gastric secretion of $\mathrm{HCl}$. An augmented histamine test. Brit. med. J., 2, 77-80. 
Lawrie, J. H., and Forrest, A. P. M. (1965). The measurement of gastric acid. Postgrad. med.J., 41, 408-417.

, Smith, G. M. R., and Forrest, A. P. M. (1964). The histamineinfusion test. Lancet, 2, 270-273.

Mason, M. C. (1968) Unpublished observations. Gut, 10, 34-38.

Multicentre Pilot Study (1967). Pentagastrin as a stimulant of maximal gastric acid response in man. Lancet, 1, 291-295. 